\title{
An intoxicated man with facial trauma
}

\author{
Colleen Birmingham - Kevin Ban · Peter Rosen · Richard Wolfe \\ Danny Davis $\cdot$ John Sakles $\cdot$ Kenny Bramwell $\cdot$ Leon D. Sanchez
}

Received: 19 January 2010/Accepted: 29 January 2010/Published online: 13 March 2010

(C) SIMI 2010

Dr. Colleen Birmingham: The chief complaint is status post battery. The patient was a 51-year-old man who was brought in by Emergency Medical Services (EMS). EMS was called because bystanders found the patient intoxicated with obvious facial trauma. The patient was combative with EMS. He told them he had been beaten, but wouldn't provide a further history. By the time he arrived at triage, he was yelling and combative. Past medical history, medications, and allergies were all unknown. On physical examination, the vital signs were: temperature $37^{\circ} \mathrm{C}$ $\left(98^{\circ} \mathrm{F}\right)$, blood pressure $196 / 130 \mathrm{mmHg}$, heart rate 96 beats/ min, respiratory rate 24 breaths/min, and oxygen saturation $100 \%$ on a $100 \% \mathrm{O}_{2}$ non-rebreather. On physical examination, he was noted to be combative, had a c-collar in place, and had the odor of alcoholic beverages on his breath. He had some facial abrasions that were bleeding, an obvious tongue laceration, and a couple of loose teeth. The lungs were clear to auscultation. There was a regular rate and rhythm. The abdomen was a little bit distended, but

C. Birmingham $(\bowtie) \cdot$ K. Ban $\cdot$ P. Rosen $\cdot$ R. Wolfe ·

L. D. Sanchez

Department of Emergency Medicine, Beth Israel Deaconess

Medical Center Harvard Medical School, Boston, MA, USA

e-mail: cbirming@bidmc.harvard.edu

J. Sakles

Department of Emergency Medicine, University of Arizona,

Tucson, USA

D. Davis

Department of Emergency Medicine, University of California, San Diego, USA

K. Bramwell

Boise, ID, USA otherwise non-tender, and he was moving all of his extremities.

Dr. Richard Wolfe: I am not even sure how you were able to get much of an examination if he was combative. I think the first issue is that you have to get this patient under control so you can assess where he is going. Do you need to go to an airway to do that, or can he just be chemically sedated to be more cooperative in order to for you to get a better sense of what is going on?

Dr. Peter Rosen: I think that there are many trauma units that would use the man's combativeness as a reason to paralyze and intubate him. But I don't think that is a good indication for active intubation, rather I think that there is a good reason, as Dr. Wolfe said, to get chemical control of this man. I personally would probably start with haloperidol. Many times I have found that once the patient is sedated from the haloperidol, your reason to intubate disappears, and you have enough cooperation from the patient that you can assess many of the things that you can't assess once you have paralyzed and intubated him. Dr. Bramwell, you are a big fan of sedation of alcoholic patients. Do you have a different methodology that you prefer?

Dr. Kenny Bramwell: My challenge with this patient is that I have had a different experience with trying to sedate alcoholics, in that I don't seem to be able to get them chemically restrained quickly enough where I feel that they are protecting their airway or, more importantly, protecting their cervical spine. If you are going to go the way of sedation, I think haloperidol or something similar to that would be very helpful because it would allow the patient to spontaneously breathe. The bigger challenge is, will this person, in a reasonable time period, be cooperative enough 
to allow you to do an examination and an expedited trauma evaluation?

Dr. Wolfe: It's funny to have to go back and revisit things like haloperidol and droperidol, which at one point our feeling was, should be put in the water. But ever since the FDA, in an ill-informed way, put a black box around droperidol, and created this unwarranted fear of neuroleptics, people are afraid to use them, and the dosages they use tend to be sub-par. Certainly in the days when we were administering $10 \mathrm{mg}$ of haldol right off the bat or $5 \mathrm{mg}$ of droperidol, the usual response time was 4-5 min. We did a study in the pre-hospital system where we actually had the paramedics administering droperidol in the field. By the time they arrived in the Emergency Department (ED), a good percentage of the patients were well sedated and in control, even though they had been combative at the time the EMS people picked them up [1]. But, I agree, if it's not working quickly enough, and you have to get control, you may have to move on.

Dr. Rosen: We did a study on haloperidol in Denver many years ago, and we found that $95 \%$ of the patients who received $10 \mathrm{mg}$ of haloperidol IV were in total control within $2 \mathrm{~min}$. Now, the one danger to haloperidol is that there are a number of patients who seem to have combined alcohol with cocaine. When you give them haloperidol, it seems to precipitate a dysrhythmic arrest [2]. I don't know whether it's the haloperidol or whether it's the cocaethylene that kicked in at that point that they received haloperidol, but I am aware of several case reports in which patients who received haloperidol and who had cocaine as well as ethanol on board had a sudden arrest. I don't think that is a reason not to use it, but it's certainly a reason to watch the patient carefully after you give the haloperidol. Dr. Davis, what are you using in San Diego for this kind of patient.

Dr. Daniel Davis: I was going to first comment that we wrestle with this in the air- medical environment, as there are important safety concerns with an uncontrolled patient in the air. At the same time, this patient is portrayed as having a small neck and some oral/airway trauma, raising concerns about rushing into this airway. I was disappointed when droperidol was removed from the helicopters as it was always our drug of choice in these situations due to its rapid onset of sedation, and general maintenance of airway protective reflexes. Ketamine may emerge as a good substitute. Many of us who study head injury feel that ketamine received a "bad rap" with early reports of a rise in intracranial pressure (ICP) with its use. It is now recognized that any rise in ICP, which generally does not seem to occur, generally reflects improvement in hemodynamics, with a rise in cerebral perfusion pressure. Would anyone else consider ketamine to be a viable option in this particular patient?

Dr. John Sakles: It's funny that you mention that because I've been going to ketamine a lot for some of these patients who need to be rapidly sedated. We have very aggressive trauma surgeons, and if you can't get patients adequately sedated in a 2 or 3 min time frame, they want the patient intubated. There is really no drug that we have that can act that rapidly. So I've gone to ketamine sometimes with these patients. If you give a small dose, you can have the patient in a cooperative state in about $60 \mathrm{~s}$. Now if you are using lorazepam or haloperidol, it usually takes a few minutes, and the trauma surgeons are usually pretty impatient with that. But if you give them a small dose of ketamine-maybe just $50 \mathrm{mg}$, not even a true induction dose, the patient just lies there and is protecting the airway, and calms down. The tone of the whole room changes in about a minute. By that time, the other drugs start to kick in. So I've used ketamine a number of times, and it's gotten me through this exact situation.

Dr. Rosen: Why don't we hear what the actual course was at this point?.

Dr. Birmingham: This patient came in right at the end of the overnight shift, a little before 7 a.m.. The overnight team did sedate the patient. He received $10 \mathrm{mg}$ of haloperidol and $2 \mathrm{mg}$ of lorazepam with a plan of imaging of his head and c-spine. He still wasn't quite sedated enough so he received four more milligrams of ativan, totaling $6 \mathrm{mg}$ of ativan. At that time, he went for CT scan of the head and c-spine. The CT head was read preliminarily as negative, but they read the c-spine as a possible C-6 fracture, but they couldn't be sure because the patient was moving and there was too much artifact. He was brought back to his bed, and the plan was to continue to sedate him for repeat imaging.

At this point the day team was caring for the patient. $\mathrm{He}$ had received a total of $15 \mathrm{mg}$ of haloperidol and $8 \mathrm{mg}$ of lorazepam for sedation. He still had copious blood in the oropharynx. The nurse was at the bedside, suctioning blood and clots from the back of the throat. He was in soft four point restraints, and he was swinging his neck and biting at his c-collar. At this point, there was concern that we were not going to be able to get him sedated enough for repeat imaging. We moved forward with the plan for a semielective intubation for imaging.

Dr. Rosen: The point I would like to make about haloperidol and many other sedatives-I believe that if you give too small of an initial dose, you get a paradoxical stimulatory effect rather than a sedating effect. So, my practice with haloperidol is to start with ten and double it every 2 min until you have complete control. I think that is 
much more likely to be successful than trying to chase the patient with small increments of haloperidol. But having said that, I can't argue with the plan here now that you have a suggestion of a c-spine fracture, which I find clinically to be rare in the presence of significant facial trauma. I don't know what the difference is in the mechanism of forces transmitted to the head and neck, and when you don't know those forces, cervical spine injury is worrisome. It would appear that without torqueing forces, with the maximum force being exerted directly through the face, cervical spine injury is rare. But, here you have a radiologic concern for a cervical spine injury. So, whatever your clinical experience is, you have to say that's something you need to worry about, and I think you have clinical reason to get total control of this patient, and I personally would be in agreement with a decision to paralyze and intubate him.

Dr. Wolfe: I just stress that you look at the total dose of a benzodiazepine here, $8 \mathrm{mg}$ in a guy who has alcohol on board, and on top of it has bleeding, so he has more of an airway risk of aspirating the blood, so that sedating strategy has created more of a problem than a benefit. I don't have a problem with a little benzodiazepine in a drunk as you start. But, frankly that's why you want to go to a neuroleptic or ketamine in this group, and not benzodiazepines.

Dr. Sakles: I agree with that. And the other problem with the benzodiazepines is the onset is just too delayed. By the time they start kicking in and as you're titrating, you are dealing with this patient for about an hour. If he has serious injuries, you are not going to be able to get any imaging done in cases like this for an hour. The benzodiazepines, in addition to having the horrible side effects, the respiratory depression and whatnot, just take too long to titrate. I think they're nowhere nearly as quick as is haloperidol.

Dr. Rosen: I agree with Dr. Sakles. The effect of the benzodiazepines that is quick is the respiratory depression-it happens almost immediately, and it's often missed if you don't watch for it. Dr. Sakles, here we have a patient with a possible c-spine fracture who is in a c-collar, who has a mouth full of blood, and who needs to be intubated. What is the approach you like the best in these patients?

Dr. Sakles: Like you, I am a little reluctant to paralyze patients like this, so what I'll usually try as my first line attempt is an awake intubation with ketamine. If you give the ketamine up front, it usually allows you to evaluate the airway: take a look and see what the anatomy is like, and I can usually get the tube in. If not, then I go ahead and paralyze the patient if I got a reasonable enough look, and I think I can get the tube in. I start with ketamine as my first line, and either get the tube, or proceed onto rapid sequence intubation (RSI).
Dr. Bramwell: On your initial look, what dose are you using for the awake intubation part phase?

Dr. Sakles: I find with these people they don't really need a full induction dose. I use about 40 or $50 \mathrm{mg}$ as the initial dose, and see what the response is. I sometimes have to repeat that so they may get another 40 or $50 \mathrm{mg}$. But I am surprised by how little ketamine is necessary to really evaluate a patient's airway like this. It's not unusual for the first 40 of $50 \mathrm{mg}$ to give you adequate sedation to take a look because they've got other drugs on board. They've got the alcohol and everything else on board, so you usually don't need a lot of ketamine.

Dr. Wolfe: Why not-because I understand this guy is going down and we need to manage his airway-but it's not something that needs to be done in $10 \mathrm{~s}$, so why not do this fiberoptically? I understand that there is bleeding going on back there, but sometimes you can sink the scope down, and as you are going to have to maintain in-line stabilization when you intubate this guy, it's going to make the oral approach potentially more difficult, and I would argue that if you have time, it might be worth trying a fiberoptic pass.

Dr. Sakles: You know the fiberoptic scope is a great tool and I've been down that road in this type of patient before, and it just doesn't work well. The problem is that the fiberoptic scope has a very small optical portion, and it just doesn't work well. It's just a little dot, like $3 \mathrm{~mm}$ or so and it's almost impossible to insert it into a patient's nose or mouth like this and not get it contaminated with blood. On top of it, you've got a patient who is not cooperative; who is moving around and it makes the procedure very difficult. I would much more likely go to a rigid videoscope than a fiberoptic scope in this circumstance. Again, the videoscope lens is a little bit more protected, it's a larger lens so it's less prone to contamination, so if I was going to use an optical devise in this patient I would go with a videoscope as opposed to a fiberscope.

Dr. Rosen: Would anyone consider a surgical airway on a patient like this to start?

Dr. Davis: I would certainly anticipate that a surgical airway is where we might end up. With that in mind, I would try to define his anterior neck anatomy before we paralyze him, which we have the option to do since it's not a crash intubation. We can prepare and gather all of the necessary equipment, and we can attempt to palpate relevant structures through the skin. We can even use ultrasound to identify peri-laryngeal structures [3]. Draw on his neck with a pen and prepare the skin in advance. That said, my primary approach would still be orotracheal intubation, ideally using a video laryngoscope. 
In my own teaching, I have emphasized trying to establish a patent airway, including the use of a nasopharyngeal airway, even with evidence of facial trauma, to ensure that we can bag-valve-mask ventilate this patient. We would make a "pig nose" and carefully insert a nasopharyngeal airway straight into the face, not upward toward the turbinate's. This increases our chances of being able to pre-oxygenate and re-oxygenate between attempts, and may buy us enough time to safely use a video laryngoscope. Pre-oxygenation is going to be an important factor for this patient.

Dr. Wolfe: Let me just ask a quick question of Dr. Birmingham. There is no Le Forte fracture in this guy? He has been imaged at this point, we have a cervical spine fracture, facial abrasions, but no major facial fracture-is that correct?

Dr. Birmingham: Not as far as we know. He had not had a maxillo-facial CT at this point, but no facial fractures as far as we could see on the head CT scan.

Dr. Wolfe: But clinically, were there any signs of major facial trauma?

Dr. Birmingham: No his face was stable, no bony crepitus. Just some abrasions and ecchymoses.

Dr. Wolfe: So you have to remember, before the advent of a lot of these toys, this was a patient where the first attempt would have been a blind nasal intubation in the ED followed by a cricothyrotomy if the nasal intubation were unsuccessful. Obviously, we were under the misconception that you couldn't orally intubate unstable c-spine fractures. In those times the blind nasopharyngeal would actually be relatively effective.

Now my point with the fiberscope, I wouldn't put the fiberscope in first. I would put the nasal tube in first, try to get as close to positioning as possible and then use the fiberscope as an enhanced visual tool at that point when you passed it.

Dr. Rosen: I've had similar experience to Dr. Sakles with the fiberoptic scope. If there are any secretions or blood, at least in my hands, they very quickly obscure the view of the fiberscope. And I've done a lot of blind nasal intubations on patients like this. Of course in the days before we believed it was safe to intubate orally, we did a lot of cricothyrotomies on patients like this because we couldn't clear the c-spine sufficiently. So, it probably doesn't matter what approach you take as long as you get control of this patient. I am very happy to hear that the modern attitude toward ketamine is that it's not contraindicated in the presence of head injury because it is a wonderful drug to get control of these patients, and it does work very rapidly, and maybe it's safer in the long run than the haldol because it doesn't seem to produce the cocaethylene dysrhythmia.

Dr. Kevin Ban: Along with deciding which approach and corresponding medications will be necessary to control the patient's airway, one must also decide who will perform the procedure. In teaching hospitals where residents often want to make the first attempt, the decision to allow a physician-in-training to perform the intubation is a mistake since there may not be a second chance to place the breathing tube. The most experienced physician should perform the procedure in very difficult patients.

It is also prudent to be conservative when managing a difficult airway. While most of us attempt direct orotracheal intubation as our first airway approach prior to using a rescue technique, it may be safest to place a bougie in the patient's trachea to be sure you can control the patient's airway.

Dr. Rosen: How did they finally intubate the patient?

Dr. Birmingham: We brought the patient into the resuscitation bay, and he was bagged initially just to make sure he could be ventilated. He received succinylcholine $120 \mathrm{mg}$ IV and etomidate $20 \mathrm{mg}$ IV. The first attempt was made by the junior resident by direct laryngoscopy with a MAC 4. There was a lot of blood in the airway, which was suctioned, but there was a big floppy epiglottis and she was not able to visualize the cords. The senior resident then took a Miller blade and took a look. She was able to see the cords, but there was a lot of mouth edema preventing her from passing the tube directly. She passed a bougie that she thought had passed through the cords, and she passed the tube over that, but it was an esophageal intubation. There was no color change, and there were epigastric stomach sounds with bagging. At that point, the attending took a look, and was not able to see anything-just a lot of edema and blood in the airway.

At that point the patient's vital signs were concerning: he was bradycardic to a heart rate of 27 beats/min. He received $2 \mathrm{mg}$ of atropine around this time, and his blood pressure went down to 73/46. The senior resident was able to place a laryngeal mask airway (LMA) after which the patient's heart rate, blood pressure and oxygen saturation all improved. He could be ventilated at that time. Anesthesia was called down with their fiberoptic scope, and they did not remove the LMA, but were able to take a look through the LMA, and were able to pass a very small endotracheal tube through the LMA in order to stabilize the airway. Surgery was called for the option of a surgical tracheostomy. The patient was brought up to the operating room and was prepared for a tracheostomy, but anesthesia was able to intubate in the operating room using a fiberoptic scope. 
Dr. Rosen: Was the videoscope used in the initial intubation attempt?

Dr. Birmingham: The glidescope was attempted after the intubation attempt with the miller blade, but the mouth was so swollen that the handle would not fit in the patient's mouth.

Dr. Rosen: I agree with Dr. Ban's earlier comment, and would submit that there are times when it is not appropriate to allow junior housestaff to start the intubation. I think this is a case where the most experienced person in the room should make the initial intubation attempt. And I say that with all due respect for the technical competency of housestaff who are often better at it than I am. But I think this is a case where senior experience is really mandatory.

I think you did a good job with a very difficult case and got control. I probably would have reached for a knife and done a surgical airway on this guy when I couldn't intubate him orally but that's just a personal preference.

Dr. Bramwell: I would think that there are a lot of clinical settings and a lot of practice settings where a surgical airway is going to be required rather than having anesthesia come down and do a fiberoptic scope, which in my mind has already been tried and failed in this setting of excessive secretions and blood. I would think that you would need to be willing and able to do a surgical airway when your one or two looks at an oropharyngeal airway have failed.

Dr. Rosen: I am a little puzzled as to why you would need a surgeon. They haven't any experience with cricothyrotomy greater than an emergency medicine physician, and in fact maybe less. I think this is not necessarily a patient who is going to need a tracheotomy, so I personally would have done a cricothyrotomy on this patient earlier but, as I said, you managed to avoid it. I think that each institution has its own protocols that it needs to follow. Most of us are going to be working in a place where we don't have ready access to surgery or to anesthesia in the ED. In many rural EDs, the best you will get is a nurse anesthetist who will have no access to special tools, and certainly no ability to do a surgical airway. I think emergency physicians need to continue to train in surgical airway expertise, and even though we do many fewer than we used to, we must still have that willingness to act, which I think characterizes the emergency physician.

Dr. Davis: A couple of other points. One, the LMA and the other extra-glottic airways are not bad options here. A new version of the LMA, the Supreme, may be especially useful to the emergency physician as a salvage device. It is essentially a non-reusable version (they don't like to call them disposable) of the pro-seal, which provides a tight seal allowing relatively good airway protection and ventilation. Because of the specific shape of the cuff, it seats itself very nicely between the base of the tongue and the epiglottis without folding over. Having this transitional airway in place may give you the option to do a cricothyrotomy under more controlled circumstances.

The second point I want to make is regarding the bradycardia that occured in this case. The pediatric experience, where vagal-mediated bradycardia often accompanies intubation attempts, has perhaps clouded our judgment a bit in adults, where we are more likely to see bradycardia related to hypoxia. I will come down hard on our residents or flight crews if bradycardia in a hypoxic patient is treated primarily with atropine rather than aggressive attempts to maintain an airway and provide adequate oxygenation. The bradycardia that accompanies hypoxia is generally not atropine responsive.

Dr. Rosen: Dr. Sanchez, do you have any final thoughts on this case?

Dr. Leon Sanchez: No I think that last point about the bradycardia is a very good point. Because I think it is a reflex that people see bradycardia, and they reach for the atropine, and atropine will not fix hypoxia. The other thing I think we should look at here is the use of ketamine because I don't think we use it as much as we should. I think there are several types of situations where ketamine might be a better induction medication than our more commonly used intubation medications.

Dr. Rosen: Good-very interesting case. Thank you Dr. Birmingham. That was a very hard airway, and I am glad I didn't have to be there to manage it.

Conflict of interest None.

\section{References}

1. Rosen CL, Ratliff AF et al (1997) The efficacy of intravenous droperidol in the prehospital setting. J Emerg Med 15(1):13-17

2. Patel MB, Opreanu M et al (2009) Cocaine and alcohol: a potential lethal duo. Am J Med 122 (1):e5-e6

3. Ma G, Davis DP et al (2007) The sensitivity and specificity of transcricothyroid ultrasonography to confirm endotracheal tube placement in a cadaver model. J Emerg Med 32(4):405-407 